Discussion Paper No. 826

\title{
SEEKING HARMONY AMIDST DIVERSITY: \\ CONSENSUS BUILDING \\ WITH NETWORK EXTERNALITIES
}

\author{
Chia-Hui Chen \\ Junichiro Ishida
}

December 2011

The Institute of Social and Economic Research

Osaka University

6-1 Mihogaoka, Ibaraki, Osaka 567-0047, Japan 


\title{
Seeking Harmony Amidst Diversity: Consensus Building with Network Externalities
}

\author{
Chia-Hui Chen*and Junichiro Ishida ${ }^{\dagger}$
}

December 16, 2011

\begin{abstract}
A group of individuals, with a potential conflict of interest, face a choice among alternatives. There is a network externality such that the chosen alternative yields value only if sufficiently many individuals get on board. Their preferences for each alternative and the benefit derived from a successfully formed network are known only privately and might vary between the players who determine whether to make their choices early or late. We characterize the equilibrium timing of adoption as well as the efficient timing which maximizes the total expected payoff. We also show that the efficient timing of adoption can be implemented by a simple fee scheme. The analysis gives an insight into why consensus is often hard-won in some societies and suggests a potential role of social norms in improving the efficiency.
\end{abstract}

\footnotetext{
*Institute of Economics, Academia Sinica. Email: chchen@econ.sinica.edu.tw

${ }^{\dagger}$ Institute of Social and Economic Research, Osaka University. Email: jishida@iser.osaka-u.ac.jp
} 


\section{Introduction}

A group of individuals, with a potential conflict of interest, face a choice among alternatives. There is a network externality such that the chosen alternative yields value only if sufficiently many individuals get on board. The group members thus need to cooperate, to some extent, to reach consensus among them to realize greater value. Consensus is often hard-won, however, because there are no coordination devices or central planners to sort out differences in opinions in many cases; as a result, consensus often needs to be built in a highly decentralized manner. With this decentralized nature, coordination failures become a real concern to be reckoned with. One potential problem is that too many individuals may take a stance early on, in order to sway others and have things in their own favor. On the flip side, it could equally be problematic if too many of them abstain and wait for others to take a stance. In either case, when the right person does not step up at the right timing, we may fail to reach consensus swiftly enough to fully reap the benefit of a network.

This type of problem abounds in many facets of our life. For instance, firms often need to choose a technology or a platform among possible alternatives. In many cases, in the presence of network externalities, only one of them will survive in the end as the standard technology: a classic example of this case is the competition of the videotape format between Sony's Betamax and JVC's VHS; more recent examples include the choice among the operating systems, wireless communication protocols, electric payment technologies, and many others. Consumers also face a similar coordination problem when they need to purchase network goods, such as telecommunication devices, personal computers (operating systems), social networking services, and video game consoles, whose value depends on and increases with the number of users. In a broader perspective, the problem can also arise in almost any circumstances in which individuals hold different opinions to various extents, but benefit from choosing the same side as others, e.g., when politicians or countries hold different political stances but want to join a larger coalition (political parties, international treaties, free trade agreements, etc) to achieve greater benefit, ${ }^{1}$ or when committee members rank candidates differently but would like to vote for the winner for some reasons.

The main purpose of the paper is to provide a synthetic illustration of the nature of inefficiency that could arise in this type of environment and a potential remedy for that. The

\footnotetext{
${ }^{1}$ Free trade agreements are often initiated by a small group of countries which set the tone for the basic framework and, if successful, later followed by other countries. For instance, the current European Union was initiated by six countries (Belgium, France, Italy, Luxembourg, the Netherlands and West Germany) as the European Coal and Steel Community, which have subsequently expanded and developed into its current form through several expansion phases. As a more recent example, the Trans-Pacific Partnership (TPP) was initiated by four countries (Chile, New Zealand, Singapore and Brunei) but has now expanded to include the US and other countries.
} 
tradeoff of our focus is simple: by taking a stance and making his intention clearer early on, one may attract more people to take his side, but loses chance to learn of what others think. To illuminate this tradeoff in the simplest manner, we consider a two-period problem faced by two individuals who must choose either one of the two alternatives, which we call "projects" for expositional clarity. In each period, the two individuals simultaneously choose whether to adopt either project or to wait and see the other's decision. A decision to take a side entails some fixed cost and is hence irreversible once it is made. They can reap the benefits of a network when both choose to work on the same project, but all the potential benefits are lost when they fail to coordinate their decisions.

The problem is compounded by the differences in their preferences. We assume that each individual's preferences, which are only privately known, could differ in two dimensions. In one dimension, individuals are heterogenous with respect to their intrinsic tastes for the projects: some individuals have a clear-cut stand on what they like and dislike (the type with strong preferences), while others are more accommodating and indifferent between the projects (the type with weak preferences). In the other, individuals also differ in the extent to which they can benefit from a successfully formed network: some derive a large marginal benefit from a network (the dependent type), while others do not derive much from it (the independent type). We assume that the type in each dimension is independent of that in the other, so that there are four different types of individual to be considered. The differences in preferences amount to the potential conflict of interest which makes the model highly strategic in nature.

We first show that, perhaps quite intuitively, the types with strong preferences adopt earlier than those with weak preferences in equilibrium. This is because a player with strong preferences can induce the other player to follow his decision by letting his intention known and thereby increasing the probability that his preferred project will be chosen. Between the two types with strong preferences, the dependent type is less inclined to adopt early because he derives a larger benefit from reaching a consensus, and so acts more cautiously to ensure that the cooperation will eventually emerge. This equilibrium allocation is, however, often not efficient, in that it does not maximize the expected total payoff, because each player considers only his own payoff and ignores the fact that his timing of adoption will also affect the other player's payoff.

To explore welfare implications of the model, we proceed to characterize the efficient allocation, which turns out to be far more complicated than the equilibrium allocation even in a simple setup like this. To characterize the efficient allocation, there are two distinct cases we need to consider, depending on the difference in the values of a network between the 
dependent and independent types. When the difference is small, the important dimension is which project to work on as a group. It is then efficient to let the types with strong preferences adopt first, followed by those with weak preferences. While this ordering could emerge in equilibrium, the equilibrium allocation can still be inefficient because it does not realize the right allocation of types between the periods, i.e., the types with strong preferences adopt in the first period either too much or too little. The inefficiency is exacerbated even more when the players are more heterogenous in productivity. In this case, the efficient allocation should assure to have the dependent types in the network, regardless of the strength of preferences. The way to achieve this is either to let the dependent types adopt first and then the independent types follow, or vice versa. As stated, though, this ordering never occurs in equilibrium because the types with strong preferences always adopt earlier.

Given that the efficient allocation cannot often be sustained in equilibrium, we then explore ways in which to restore the efficient allocation. We obtain two results on this. First, we show that the (constrained) efficient allocation can be achieved by imposing fees on individuals adopting in different periods, without resorting to more complicated mechanisms. The appropriately designed fee scheme, which reallocates the costs and benefits among the types, corrects not only which type of individual will step forward to be the leader but also the probability that each type will step forward. We argue that this simple property is critical given the spirit of the problem that consensus is often built in a decentralized manner: even when an almighty central planner can achieve a better allocation by designing an incentive compatible mechanism and implementing it in a more complex and dictatorial manner, such an option is rarely available or highly costly in the class of problems under consideration. ${ }^{2}$ In contrast, our solution suggests that the efficiency can be restored simply by placing more or less burden on those who speak up first, which is supposedly much easier to implement. As one possibility, social norms may play an important role in reallocating the costs between the leader and the follower.

Second, the current analysis also clarifies when it is more desirable to place an additional cost on the leader. We find that it is welfare-improving to impose a positive fee on the leader either when the players are relatively homogeneous in productivity or when they are diverse in both productivity and preferences. This result suggests that the norm which shifts more burden to the leader works well in a wide range of circumstances, thereby lending support to a prevalent social norm that leaders should assume more responsibility and accept the

\footnotetext{
${ }^{2}$ Our proposed fee scheme achieves a constrained optimum as it only allocates the right proportion of types to each period but does not resolve the coordination problem. In this sense, the central planner can do better by designing an appropriate direct mechanism and assigns the players to each period in a way to avoid coordination failures.
} 
burden of accountability. Our analysis reveals, however, that this type of social norm does not always improve welfare. When the players are diverse only in productivity but have more homogeneous preferences, they are reluctant to speak up and tend to abstain too often in equilibrium. In this case, it is desirable to give an extra reward to the leader to encourage more people to step up to lead the way.

Our analysis is closely related to studies on voting where the timing of expressing one's opinion could be decisive. In the literature, considerable attention has been devoted to studying how simultaneous voting and sequential voting affect electoral outcomes (see e.g. Dekel and Piccione (2000), Battaglini (2005), Battaglini, Morton, and Palfrey (2007)). In particular, Callander $(2007,2008)$ considers the situation in which voting is sequential and voters prefer to vote for a winner. ${ }^{3}$ The environment is similar to that under consideration here, but in traditional sequential voting models, voters cast their votes in an exogenously fixed order, whereas in ours, voters strategically choose when to cast their votes. ${ }^{4}$

The timing of expressing one's opinion is also critical in the legislative branch of government. In the US Congress, for instance, legislators need to determine not only how to vote, but also when to announce their position on the issue during the weeks or months before the final vote. This type of strategic interaction is obviously not a unique phenomenon in the US, as it can be observed virtually in any legislative process. Legislators are generally concerned with the opinions of constituents, interest groups, and party leaders, and thus need to weigh the costs and benefits when they decide when to make their opinions clear. Box-Steffensmier, Arnold, and Zorn (1997) and Caldeira and Zorn (2004) provide evidence showing that legislators whose constituencies, interest group influences, and partisanship are in alignment announce their positions earlier, and those with conflicting or ambiguous signals delay their announcements. Provided that the former case of aligned interests corresponds to the types with strong preferences, we argue that this observation is in line with our main results.

This paper is also related to the literature on the diffusion of technologies with network externalities. Farrell and Saloner (1986) and Farrell and Shapiro (1993) consider the timing that different groups of people choose to switch from an old technology to a new technology. Farrell and Shapiro (1993) further study the situation in which different groups initially use incompatible technologies and analyze which group switches first in equilibrium. This paper

\footnotetext{
${ }^{3}$ Callander shows that a desire to conform with the majority induces momentum and bandwagons, two phenomena commonly observed in real sequential elections.

${ }^{4}$ Ottaviani and Sorensen (2001) consider decision making in a committee consisting of members who wish to appear well informed and analyze the optimal order of speech (who to speak up first). In this work, however, the order is also exogenously imposed form outside.
} 
goes one step further by examining how users with different characteristics choose between different new technologies and the timing to adopt them.

The remainder of this paper is organized as follows. Section 2 describes the model. Section 3 characterizes the equilibrium timing of adoption. Section 4 characterizes the efficient timing of adoption and makes a point that the equilibrium timing is often inefficient due to network externalities. Section 5 shows that one can restore the efficient outcome by a simple fee scheme. Finally, Section 6 concludes.

\section{Model}

Environment: Two risk-neutral players, denoted by $i=1,2$, are asked to make a choice between two projects, $a$ and $b$, over two periods. In each period, each player chooses his stance $s_{i, t} \in S_{t}$ where $S_{1}=\{a, b, A\}$ and $S_{2}=\{a, b\}$. For expositional purposes, we say that a player "adopts" when either $s_{i, t}=a$ or $s_{i, t}=b$, while he "abstains" when $s_{i, t}=A .{ }^{5}$ The decision is irreversible once a player adopts in the first period, one way or the other, i.e., $s_{i, 1}=s_{i, 2}$ if $s_{i, 1}=a$ or $s_{i, 1}=b$. A network is formed successfully and the chosen project yields value only if $s_{1,2}=s_{2,2}$, i.e., the two players agree to take the same side.

Payoffs: The payoff for player $i$ is given by

$$
u_{i}=\left[v_{i}-c\left(\tilde{s}_{i}, s_{i, 2}\right)\right] x,
$$

where $x=1$ if a network is formed successfully and $x=0$ otherwise. Each player's preferences are characterized by $\left(v_{i}, \tilde{s}_{i}\right)$, which captures two-dimensional preference heterogeneity in this context. We assume that both $v_{i}$ and $\tilde{s}_{i}$ are the player's private information and not observable to the other player.

First, the value of a network may vary between the players, where some derive a larger marginal benefit from a network while others derive a smaller benefit. For expositional purposes, we refer to the former as the "dependent" type and to the latter as the "independent" type. The difference in the values captures one source of heterogeneity between the players which yields at least two distinct (and possibly conflicting) ways to interpret, depending on the underlying context: in one interpretation, the dependent type can be regarded as the one who needs to rely more on the network, hence the label dependent, whereas the independent type is the one who is "large" enough by himself and cares less about what others do; in the

\footnotetext{
${ }^{5}$ We assume that the option of abstention is available only in the first period. This assumption is made purely to simplify the exposition and absolutely inessential because each player possesses veto power in this setup. To be more precise, a player would abstain in the second period only when there is a leader who chose the project he dislikes in the first period. In this case, however, the player can reject the proposal by simply choosing the other project.
} 
other, the dependent type can also be the more productive type who can exploit more from the existing network. The probability that a player is dependent is $p$, i.e.,

$$
\operatorname{Prob}\left(v_{i}=v_{H}\right)=p, \operatorname{Prob}\left(v_{i}=v_{L}\right)=1-p
$$

Second, the players may also differ in the strength of preferences with respect to the project, where each player's preferred project is denoted by $\tilde{s}_{i} \in\{a, b, n\}$. A player has a firm stand on what he likes and dislikes when $\tilde{s}_{i}=a$ or $\tilde{s}_{i}=b$ (the type with strong preferences), whereas he is fairly neutral and indifferent between the positions when $\tilde{s}_{i}=n$ (the type with weak preferences). The strength of preferences determines the cost of working on the project, where

$$
c(a, a)=c(b, b)=0, c(n, a)=c(n, b)=\frac{d}{2}, c(a, b)=c(b, a)=d>0 .
$$

That is, a player with strong preferences incurs a disutility of $d$ (zero) when he works on the project he dislikes (likes); a player with weak preferences incurs a disutility of $\frac{d}{2}$ no matter which project is chosen. ${ }^{6}$ We also assume that $v_{L}>\frac{d}{2}$ to ensure that for each type, there exists a project on which he can cooperate with the other player and derives a positive payoff. Finally, the probability distribution of each type is given by

$$
\operatorname{Prob}\left(\tilde{s}_{i}=a\right)=\operatorname{Prob}\left(\tilde{s}_{i}=b\right)=\frac{q}{2}, \operatorname{Prob}\left(\tilde{s}_{i}=n\right)=1-q .
$$

Each player's strength of preferences is independent of the benefit derived from a network $v_{i}$. There are hence four types of player: the dependent type with strong preferences (DS), the dependent type with weak preferences (DW), the independent type with strong preferences

\begin{tabular}{|c|c|c|}
\hline & $\begin{array}{c}\text { Strong preferences } \\
q\end{array}$ & $\begin{array}{l}\text { Weak preferences } \\
1-q\end{array}$ \\
\hline $\begin{array}{l}\text { Dependent } \\
p\end{array}$ & $p q$ & $(1-q) p$ \\
\hline $\begin{array}{l}\text { Independent } \\
\qquad 1-p\end{array}$ & $q(1-p)$ & $(1-q)(1-p)$ \\
\hline
\end{tabular}
(IS), and the independent type with weak preferences (IW). The distribution of player type is summarized in the following table.

Interpretation: The set of parameters $\left(v_{H}, v_{L}, d, p, q\right)$ totally characterizes the nature of the underlying environment. Among them, our primary focus is on two parameters, which

\footnotetext{
${ }^{6}$ We specify that $c(n, a)=c(n, b)=\frac{d}{2}$ in order to isolate the unintended "productivity effect". In a fully symmetric environment where the project is chosen randomly, the ex ante expected cost becomes independent of the strength of preferences. This property is important when we characterize the efficient allocation.
} 
we call the productivity dispersion $\Delta_{v}:=v_{H}-v_{L}$ and the preference (ideological) dispersion $q$. First, the productivity dispersion measures the degree of heterogeneity among the players with respect to the productivity, ${ }^{7}$ which seems to play a critical role in the case of technology adoption or any other cases in which the players differ greatly in the extent to which they can benefit from a network. ${ }^{8}$ On the other hand, the preference dispersion measures how likely a given player has a certain stance about the direction of the project, one way or the other, and hence the severity of the potential conflict of opinions. The preference dispersion plays a critical role in political situations, but is likely to be small when the players share some goal, such as profit maximization, and care more about the outcome (what is achieved) rather than the process (how it is achieved). As we will see below, these two parameters have a profound impact on the socially efficient timing of adoption, thereby yielding critical welfare and policy implications.

Timing: To sum up, the timing structure of the game is as follows.

1. Nature randomly draws $v_{i}$ and $\tilde{s}_{i}$ for $i=1,2$.

2. In the first period, each player simultaneously makes his choice $s_{i, 1}$. The choice is observable to the other player.

3. In the second period, a player who has abstained in the first period, i.e., $s_{i, 1}=A$ makes his choice $s_{i, 2}$ once again.

4. The payoffs are realized.

We seek for symmetric equilibria of this game in which the two players adopt the same strategy.

\section{The Equilibrium Timing of Adoption}

This section analyzes the equilibrium timing of adoption. The strategies of this game consist of: (i) the type-contingent probability distribution over the first-period choice; (ii) that over the second-period choice given the other player's first period choice. Instead of describing the equilibrium timing in terms of these strategies, which can be quite cumbersome, we employ an

\footnotetext{
${ }^{7}$ As mentioned above, however, it is not clear without any concrete context which one $\left(v_{i}=v_{H}\right.$ or $\left.v_{i}=v_{L}\right)$ is the more productive type, and the interpretation of this parameter should hence be made with caution.

${ }^{8}$ In contrast, we do not place much emphasis on $p$. Aside from the fact that this parameter does not easily admit an economically relevant interpretation, its role is qualitatively similar to that of $\Delta_{v}$, though not completely identical: the productivity dispersion becomes less of a factor as $\Delta_{v}$ decreases, which roughly corresponds to the case where $p$ is close to either zero or one.
} 
alternative way of characterizing the timing: any equilibrium of this game can be thoroughly described by the order of adoption across the types and $\left(r_{1}, r_{2}\right)$, where

- $r_{1}$ : the unconditional probability that a player adopts in the first period;

- $r_{2}$ : the unconditional probability that a player adopts and follows the choice made by the other player in the second period.

For clarity, we refer to the set of the order and the probabilities $\left(r_{1}, r_{2}\right)$ as the timing of adoption. The key to this representation is that if one type is indifferent between the two choices, then all the other types listed higher (lower) in the order have a strict incentive to adopt (abstain). Exploiting this feature, the order and the unconditional probabilities $\left(r_{1}, r_{2}\right)$ are all we need to characterize the equilibrium allocation which can straightforwardly be computed from the primitives of the model (see Appendix B for more detail).

We start with the second-period problem, which is meaningful only when at least one agent chooses to abstain in the first period. There are two cases we need to consider. First, if both abstain, i.e., $s_{1,1}=s_{2,1}=A$, no additional information is revealed. One can then easily check that the following constitutes a Bayesian Nash equilibrium of this static game: (i) the types with strong preferences choose $s_{i, 2}=\tilde{s}_{i}$; (ii) the types with weak preferences randomize between $a$ and $b$, each with equal probability. ${ }^{9}$ If only one player adopts in the first period, the choice for the other player in the second period is either to follow the choice or to deviate from it. Player $i$ follows the choice made by the other player if

$$
v_{i}-c\left(\tilde{s}_{i}, s_{-i, 1}\right) \geq 0, i \neq-i
$$

Given this, we now proceed to the first-period problem, where each player must decide whether to adopt or to abstain and hopefully wait for the other player to lead the way. Note first that there is clearly no incentive for a player with strong preferences to choose the project he dislikes, so that if he adopts in the first period, he always chooses the project he likes. ${ }^{10}$ We also suppose that whenever a player with weak preferences chooses to adopt in the first period, he randomizes and chooses each project with equal probability. It then follows from these that the other player is expected to choose each project with probability $r_{1} / 2$. If $v_{L}-d \geq 0$, which implies that even the independent type finds it profitable to work

\footnotetext{
${ }^{9}$ Since this is effectively a coordination game, there is also a pure-strategy equilibrium where all the indifferent players coordinate on one side. Given the spirit of decentralized consensus building, however, we assume that such coordination is either impossible or prohibitively costly, so that if a player who is indifferent between the two projects chooses to take a stance, he has no choice but to randomize and choose each with probability one half.

${ }^{10}$ If a network is formed on the project a player likes, the payoff is $v_{i}$; if on the project he dislikes, it is $v_{i}-d$. Choosing the project he dislikes is hence weakly dominated.
} 
on the project he dislikes, then a player waiting until the second period will certainly choose whichever the project chosen by the other player in the first period, if any, and so $r_{2}=1-r_{1}$. Otherwise, a player waiting until the second period chooses a different project if the project chosen by the other player in the first period is unfavorable, and hence $r_{2}<1-r_{1}$.

Given this and $\left(r_{1}, r_{2}\right)$, if a player chooses to adopt in the first period, the probability that a network forms successfully is $\frac{r_{1}}{2}+r_{2}$. If a player chooses to abstain, the other player adopts in the first period with probability $r_{1}$ and in the second period with the remaining probability. In each case, the probability that the other player chooses the project player $i$ likes is one half. ${ }^{11}$ It then follows that a player with strong preferences chooses to adopt in the first period only if

$$
\left(\frac{r_{1}}{2}+r_{2}\right) v_{i} \geq\left(\frac{r_{1}}{2}+\frac{1-r_{1}}{2}\right) v_{i}+\frac{r_{1}}{2} \max \left(v_{i}-d, 0\right) .
$$

Similarly, a player with weak preferences chooses to adopt in the first period only if

$$
\left(\frac{r_{1}}{2}+r_{2}\right)\left(v_{i}-\frac{d}{2}\right) \geq\left(\frac{r_{1}}{2}+\frac{1-r_{1}}{2}+\frac{r_{1}}{2}\right)\left(v_{i}-\frac{d}{2}\right) .
$$

Notice that both of the types with weak preferences face the same incentives which depend only on $\left(r_{1}, r_{2}\right)$.

We denote the equilibrium values of $\left(r_{1}, r_{2}\right)$ by $\left(r_{1}^{*}, r_{2}^{*}\right)$. The following two lemmas prove several properties of $\left(r_{1}^{*}, r_{2}^{*}\right)$ which will be useful for the subsequent analysis. Lemma 1 shows that if a player chooses to abstain, then he will always follow the choice made by the other agent in the first period, if any, so $r_{1}^{*}+r_{2}^{*}=1$. It is thus implied that if working on the project he dislikes yields a negative payoff to a player, then in equilibrium, he never abstains in the first period.

Lemma 1 In equilibrium, $r_{1}^{*}+r_{2}^{*}=1$.

Proof: If $r_{1}^{*}+r_{2}^{*}<1$, it is implied that among those who abstain in the first period, there exists some type that always chooses the project he likes no matter which project the other player chooses in the first period. This type cannot be the types with weak preferences because it is always optimal for these types to agree with the choice made in the first period when $v_{L} \geq \frac{d}{2}$. Moreover, we can show that it cannot be the types with strong preferences either. To see this, suppose that, for a type with $v_{i}$ and with strong preferences', it is optimal

\footnotetext{
${ }^{11}$ Note that among the types with a strong preference, one half of them prefers project $a$ and the other half prefers $b$. Also, by assumption, the types with a weak preference randomize and choose each project with equal probability. Therefore, for any type distribution, the probability that the other player chooses the project player $i$ likes is one half.
} 
to wait and choose the project he likes, regardless of the choice made in the first period. This means that $v_{i}<d$ and

$$
\left(\frac{r_{1}^{*}}{2}+r_{2}^{*}\right) v_{i} \leq \frac{1}{2} v_{i} \Leftrightarrow \frac{r_{1}^{*}}{2}+r_{2}^{*} \leq \frac{1}{2}
$$

If $\frac{r_{1}^{*}}{2}+r_{2}^{*}<\frac{1}{2}$, all the other types are strictly better off by abstaining in the first period. Since there must be some type who adopts in the first period in equilibrium, $\frac{r_{1}^{*}}{2}+r_{2}^{*}=\frac{1}{2}$.

Now notice that: (i) the unconditional probability that a player adopts in the first period is $r_{1}^{*}$; (ii) the unconditional probability that a player abstains and chooses the project he likes in the second period is $2\left(1-r_{1}^{*}-r_{2}^{*}\right){ }^{12}$ Since $\frac{r_{1}^{*}}{2}+r_{2}^{*}=\frac{1}{2}, r_{1}^{*}+2\left(1-r_{1}^{*}-r_{2}^{*}\right)=1$, meaning that only the types with strong preferences exists, a contradiction.

This equilibrium property substantially simplifies the analysis, as (1) and (2) can now be written as

$$
\begin{gathered}
\left(1-r_{1}\right) v_{i} \geq r_{1} \max \left(v_{i}-d, 0\right), \\
1 \geq 2 r_{1},
\end{gathered}
$$

respectively. Define

$$
R_{i}:=\frac{v_{i}}{v_{i}+\max \left(v_{i}-d, 0\right)},
$$

as the threshold probability, with which (3) is reduced to $R_{i} \geq r_{1}$. Note that $R_{L} \geq R_{H}>0.5$.

Next, Lemma 2 shows that the probability that a player adopts in the first period is at least as large as the probability that he abstains and waits until the second period.

Lemma 2 In equilibrium, $r_{1}^{*} \geq r_{2}^{*}$, i.e., $r_{1}^{*} \geq \frac{1}{2}$.

Proof: Suppose, on the contrary, that $r_{1}^{*}<r_{2}^{*}$. By Lemma $1, r_{2}^{*}=1-r_{1}^{*}$, so a player with weak preferences obtains a higher payoff by adopting in the first period: $\left(\frac{r_{1}^{*}}{2}+r_{2}^{*}\right)\left(v_{i}-\frac{d}{2}\right)>\left(r_{1}^{*}+\frac{r_{2}^{*}}{2}\right)\left(v_{i}-\frac{d}{2}\right)$. The same also holds for a player with strong preferences: $\left(\frac{r_{1}^{*}}{2}+r_{2}^{*}\right) v_{i}>\left(\frac{r_{1}^{*}}{2}+\frac{r_{2}^{*}}{2}\right) v_{i}+\frac{r_{1}^{*}}{2} \max \left(v_{i}-d, 0\right)$. So in equilibrium, $r_{2}^{*}=0$, a contradiction.

With the help of Lemmas 1 and 2, we can characterize the equilibrium timing of adoption. If we list type $i$ ahead of type $j$, it means either (a) that type $i$ adopts in the first period with probability one and type $j$ adopts with probability $\alpha \in[0,1]$, or (b) that type $i$ adopts in

\footnotetext{
${ }^{12} 1-r_{1}^{*}-r_{2}^{*}$ is the probability that a player abstains and disagrees with the choice made by the other player. Since there is equal probability that the other player chooses the project he likes, the probability is multiplied by two to obtain $2\left(1-r_{1}^{*}-r_{2}^{*}\right)$.
} 
the first period with probability $\alpha \in[0,1)$ and type $j$ adopts with probability zero. In either case, therefore, type $i$ adopts no later than type $j$ even in the ex post sense.

Proposition 1 The equilibrium order of adoption is (i) IS (ii) $D S$ (iii) IW/DW, where

$$
r_{1}^{*}= \begin{cases}\frac{1}{2} & \text { if } q \in\left[0, \frac{1}{2}\right] \\ q & \text { if } q \in\left(\frac{1}{2}, R_{H}\right] \\ R_{H} & \text { if } q \in\left(R_{H}, \frac{R_{H}}{1-p}\right] \\ (1-p) q & \text { if } q \in\left(\frac{R_{H}}{1-p}, \frac{R_{L}}{1-p}\right] \\ R_{L} & \text { if } q \in\left(\frac{R_{L}}{1-p}, 1\right],\end{cases}
$$

Proof: The equilibrium order of adoption is evident from (3) and (4). See Figure 1 in Appendix B for the computation of $r_{1}^{*}$.

The order characterized in Proposition 1 is fairly intuitive. As we have already seen, the types with weak preferences face exactly the same incentives and are ranked in the same order. Moreover, these types feel indifferent between the two projects, and the expected benefit from adopting first and inducing the other to follow is not as much. Between the types with strong preferences, the dependent type derives more benefit from a network, so he acts more cautiously to make sure that a network will eventually emerge, one way or the other, which can be seen from the fact that $R_{L} \geq R_{H}$. By adopting in the first period, a player expects that the probability of the two players agreeing on the same project is $\frac{r_{1}}{2}+r_{2}$; by adopting in the second period, a player expects the probability to be $r_{1}+\frac{r_{2}}{2}$. Since $r_{1} \geq \frac{1}{2}$, the probability is larger if a player adopts in the second period, meaning that the incentive to abstain in the first period is stronger for the dependent type than for the independent type.

\section{The Efficient Timing of Adoption}

In this section, we characterize the efficient timing of adoption under the restriction that decisions are made in a totally decentralized manner. More precisely, the social planner here can only dictate the probability that each type adopts in each period, but is not equipped with a tool to resolve coordination failures. This notion of constrained efficiency yields implications of more practical importance, because it could be implemented by a simple, and more realistic, institution as we will discuss in the next section. ${ }^{13}$

\footnotetext{
${ }^{13}$ While the fully efficient allocation can be implemented by an incentive-compatible direct mechanism, it is in practice not easy to find a vehicle though which to achieve this. In contrast, as we will see in the next section, the constrained efficiency can be implemented by simply imposing fees contingent on the timing of adoption.
} 
As it turns out, the characterization of the efficient timing is much more complicated than that of the equilibrium timing. To obtain clear-cut results, we make two assumptions to restrict our attention to cases that are more relevant and capture the spirit of the current analysis.

Assumption 1 Any of the four probabilities, $p q, q(1-p), p(1-q)$, and $(1-p)(1-q)$, does not exceed the sum of any other two probabilities.

Assumption $2 v_{L} \geq d$.

Assumption 1 indicates that the types are reasonably evenly distributed, and none of them is hence negligible. With some computation, one can show that a sufficient condition for the assumption is $p, q \in\left(\frac{3-\sqrt{5}}{2}, \frac{\sqrt{5}-1}{2}\right)$, whereas a necessary condition is $p, q \in\left(\frac{1}{3}, \frac{2}{3}\right)$. With Assumption 2, we restrict our attention to the case where the value of a network is so large that if only one player adopts in the first period, the other player will always follow the first player's decision, i.e., $r_{1}+r_{2}=1$.

In this problem, the social planner assigns the probability for each type to adopt in each period. This can be done in the same manner as we characterize the equilibrium timing: the social planner's problem is effectively to determine the order of adoption across the four types and $r_{1}$, i.e., the unconditional probability that a player adopts in the first period. ${ }^{14}$ The way we characterize the efficient timing is as follows. First, we fix $r_{1}$ and find the efficient order of adoption for each given $r_{1}$. We then later shift $r_{1}$ to find the efficient level, which we denote by $r_{1}^{* *}$, which maximizes the expected total payoff. Note that, given the order and $r_{1}^{* *}$, the efficient allocation can be derived straightforwardly from the type distribution characterized by $p$ and $q$, as in the case of the equilibrium allocation.

Lemma 3 Define $\delta:=\frac{d}{4 \Delta_{v}}$. The efficient order of adoption is A: (i) $D S$ (ii) $D W$ (iii) IS (iv) IW for $r_{1} \in\left(0, \frac{1}{2}-\delta\right)$,

$B$ : (i) $D S$ (ii) IS (iii) $D W$ (iv) IW for $r_{1} \in\left(\frac{1}{2}-\delta, \frac{1}{2}\right)$,

$C$ : (i) IS (ii) DS (iii) IW (iv) DW for $r_{1} \in\left(\frac{1}{2}, \frac{1}{2}+\delta\right)$,

$D$ : (i) IS (ii) IW (iii) $D S$ (iv) $D W$ for $r_{1} \in\left(\frac{1}{2}+\delta, 1\right)$.

Proof: See Appendix A.

The lemma indicates that there are four distinct candidates for the efficient order, labeled as A, B, C, and D, in this setup. In fact, as we will see shortly, any of the four orders can be

\footnotetext{
${ }^{14}$ Since $v_{L} \geq d$ and $r_{1}+r_{2}=1$ always holds, $r_{1}$ alone is a sufficient statistic.
} 
efficient under some conditions. This fact suggests that the welfare properties of the model are much more delicate and complicated compared to the equilibrium properties that are quite simple.

With this lemma, we can now characterize the efficient timing of adoption. We start with the case where the productivity dispersion is relatively small (a small $\Delta_{v}$ ), so that only regions $\mathrm{B}$ and $\mathrm{C}$ in Lemma 3 are relevant.

Proposition 2 Suppose $\Delta_{v}<\frac{d}{2}$. Then,

1. if $q>\frac{1}{2}, r_{1}^{* *} \in\left(\frac{1}{2}, q\right]$, and the efficient order is (i) IS (ii) $D S$ (iii) $I W$ (iv) $D W$;

2. if $q<\frac{1}{2}, r_{1}^{* *} \in\left[q, \frac{1}{2}\right)$, and the efficient order is (i) $D S$ (ii) IS (iii) $D W$ (iv) IW;

3. if $q=\frac{1}{2}, r_{1}^{* *}=\frac{1}{2}$, and the efficient order is (i) $D S / I S$ (ii) $D W / I W$.

Proof: See Appendix A.

The proposition characterizes the efficient timing of adoption when $\delta>\frac{1}{2}$. Given that the productivity dispersion is small, the important dimension necessarily becomes the preference dispersion, and the focus of attention is on how to choose the right project between the two. It is hence efficient to let the types with strong preferences adopt first, followed by the types with weak preferences, in any event. Given this, the next priority is to have the dependent types in a successful network, which depends largely on the likelihood of a player having strong preferences. When $q>\frac{1}{2}$ and the preference dispersion is large, it is efficient to let more people express their preferences early on $\left(r^{* *}>\frac{1}{2}\right)$. Since there is more likely to be a leader to show which way to go, the dependent type with strong preferences should take a back seat to the corresponding independent type, so that he can follow the leader's choice to be in a successful network. When $q<\frac{1}{2}$, on the other hand, it is efficient to let less people express their preferences $\left(r^{* *}<\frac{1}{2}\right)$. Now that there is more likely to be a follower, the dependent type with strong preferences should step up first to allow the player to follow his choice.

We now turn to the case where the productivity dispersion is relatively large. ${ }^{15}$ In this case, the focus of attention shifts to having the dependent types in a successful network.

Proposition 3 Given $p \in\left(\frac{1}{3}, \frac{2}{3}\right),{ }^{16} q$, and $d$, there exist $\bar{\Delta}_{v}, \bar{q}$, and $\underline{q}$ such that for $\Delta_{v}>\bar{\Delta}_{v}$, 1. if $q \in\left(\bar{q}, \frac{2}{3}\right), r_{1}^{* *} \in\left(0, \frac{1}{2}-\delta\right)$ and the efficient order is (i) $D S$ (ii) $D W$ (iii) IS (iv) $I W$; 2. if $q \in\left(\frac{1}{3}, \underline{q}\right), r_{1}^{* *} \in\left(\frac{1}{2}+\delta, 1\right)$ and the efficient order is (i) IS (ii) $I W$ (iii) $D S$ (iv) $D W$.

\footnotetext{
${ }^{15}$ Aside from the fact that $\Delta_{v}$ is large enough, this also requires $q$ to be sufficiently close to one half, because the effect of $\Delta_{v}$ would be diminished when $p$ is close to either zero or one.

${ }^{16} p, q \in\left(\frac{1}{3}, \frac{2}{3}\right)$ is a necessary condition for Assumption 1 .
} 
Proof: See Appendix A.

When the productivity dispersion is large, the first priority is to assure to have the dependent types in a successful network. The efficient order to achieve this is either that the dependent types adopt before the independent types or vice versa, regardless of the strength of preferences. The dependent types should adopt early when there is a greater possibility of attracting a follower, i.e., a small $r_{1}^{* *}$ (region $\mathrm{A}$ in lemma 3). By the same token, the dependent types should adopt late when there is a greater probability of having a leader, i.e., a large $r_{1}^{* *}$ (region $\left.\mathrm{D}\right)$.

The deciding factor between the two cases is therefore which one, a small $r_{1}^{* *}$ (case 1) or a large $r_{1}^{* *}$ (case 2 ), is less likely to have the types with strong preferences work on the project they dislike. Rough intuition for the proposition goes as follows. When the preference dispersion is large, i.e., $q \in\left(\bar{q}, \frac{2}{3}\right)$, more of the types with strong preferences are inevitably allocated to the second period. If the likelihood of having a leader, i.e., $r_{1}^{* *}$, is large, that also increases the likelihood that a follower must work on the project he dislikes. As such, therefore, it is relatively costly to have a larger $r_{1}^{* *}$ and allocate more people to the first period when the preference dispersion is relatively large. A similar argument can be applied to show that case 2 emerges as the socially efficient allocation when the preference dispersion is relatively small.

The equilibrium timing of adoption derived in Section 3 is often different from the efficient timing characterized in Section 4, because each player does not take into account the impact of his move on the other player's payoff when he decides when to adopt. To see this, note that the timing choice in this context is a strategic substitute, where the value of adopting in the first period is decreasing in $r_{1}$. When a player moves from the second period to the first, therefore, it lowers the expected payoff for the types who adopt in the first period and raises that for those who adopt in the second. These costs and benefits do not necessarily coincide at the equilibrium level $r_{1}^{*}$.

Proposition 4 If $\Delta_{v}<\frac{d}{2}, r_{1}^{* *} \leq r_{1}^{*}$. Moreover, there exists $\bar{q} \in\left(\frac{1}{2}, \frac{v_{H}}{2 v_{H}-d}\right]$ such that $r_{1}^{* *}=r_{1}^{*}$ if and only if $q \in\left[\frac{1}{2}, \bar{q}\right]$.

Proof: See Appendix A.

Proposition 5 Given $p \in\left(\frac{1}{3}, \frac{2}{3}\right)$ and $d$, there exist $\bar{\Delta}_{v}, \bar{q} \geq \frac{1}{2}$, and $\underline{q} \leq \frac{1}{2}$ such that given $\Delta_{v}>\bar{\Delta}_{v}$ 
1. if $q \in\left(\bar{q}, \frac{2}{3}\right), r_{1}^{* *}<r^{*}$;

2. if $q \in\left(\frac{1}{3}, \underline{q}\right), r_{1}^{* *}>r^{*}$.

Proof: See Appendix A.

These propositions indicate that the equilibrium allocation is largely inefficient, though not always, and moreover that $r_{1}^{*}>r_{1}^{* *}$ holds for a wide range of parameter values, meaning that too many people tend to adopt in the first period. This is mainly due to the presence of the types with strong preferences: for those players, the benefit of adopting in the first period is excessively strong because they can increase the probability of attracting other people to follow and having things in their favor by doing so. As a result, we tend to have too many leaders, with too few followers, generating excessive congestion in the first period.

\section{Implementation of the Efficient Timing of Adoption}

The results presented thus far illuminate inherent difficulties associated with consensus building in the presence of network externalities. The equilibrium timing of adoption can be inefficient on two distinct levels. First, $r_{1}^{*}$ is either too high or too low, thereby resulting in excessive congestion in either period. Second, the order of adoption can also be wrong, especially when the productivity dispersion is sufficiently large. These results provide an insight into why we often end up with a seemingly inefficient consensus or standard when it is built up in a totally decentralized manner: for instance, it is well known that the standard technologies are often not the most technically advanced (Arthur, 1994; Lee et al., 1995). In many cases, we cannot expect a right person to step up at the right timing in the presence of network externalities.

Given that decentralized consensus building is often inefficient, a natural question is then whether there is any feasible way to restore the efficient outcome. To this end, we consider the following simple fee scheme: a player must incur a cost $c_{1}$ if adopting in the first period, and $c_{2}$ if adopting in the second. ${ }^{17}$ The fees are collected by a financial institution, which reimburses each player half of its expected revenue from fee collection in advance. It is important to note that what is referred to as a fee here should be interpreted broadly: while it certainly includes monetary transfers, it can also refer to psychological costs or benefits of becoming a leader in a specific context. Without loss of generality, we assume that at least one of $c_{1}$ and $c_{2}$ is 0 : let $c_{1}=c$ and $c_{2}=0$ for normalization. As we will show next, despite the fact that the conflict of interests arises from two-dimensional heterogeneity in preferences,

\footnotetext{
${ }^{17}$ We assume that the players do not have any budget constraints.
} 
this simple fee scheme can implement the efficient timing of adoption when the fee $c$ is set at an appropriate level.

Proposition 6 For any given set of parameters $\left(v_{L}, v_{H}, d, p, q\right)$, the efficient timing can be implemented by imposing a fee $c$ on players adopting in the first period. Moreover, $c>0$ if $r_{1}^{*}>r_{1}^{* *}$ and $c<0$ if $r_{1}^{*}<r_{1}^{* *}$.

The result suggests that the ex post reallocation of costs and rewards through the fee scheme could influence the way consensus emerges in a group. A positive fee should be placed on early adopters when $r_{1}^{*}$ is too high, i.e., $r_{1}^{*}>r_{1}^{* *}$ whereas a negative fee should be placed when $r_{1}^{*}$ is too low. By changing the expected share of costs and rewards, one can design a scheme which alleviates the coordination problem, and realizes the efficient outcome in the absence of proper coordination devices.

In reality, when a group of people engage in a project, the costs and benefits are not necessarily allocated evenly among them; moreover, the allocation often depends on the timing of adoption, even without explicit ex ante agreements. Among many possibilities, we focus on social norms which often provide a way to reallocate the costs and benefits within a group. For instance, there is a strong belief that a leader must assume more responsibility and accept the burden of accountability: the leader is expected to take an aggressive role in recruiting and motivating fellow members in initiating a project, and to be the one to take the blame whenever things go wrong. "The Buck Stops Here," the well-known sign on the desk of Harry S. Truman, exemplifies this spirit. The leadership literature also emphasizes that one of the most important qualities to be held by a leader is self-sacrifice (Conger and Kanungo, 1998). This type of social norms or expectations certainly affects one's incentive to step forward to lead the way, and when the incentive to step forward is excessively strong, that could correct inefficiencies inherent in decentralized consensus building.

The allocation of costs and benefits through social norms might be harder in the context of technology adoption (either by firms or consumers), but there may still be a mechanism to allocate the costs and benefits between leaders and followers. In developing a new technology standard, pioneering firms typically need to invest more and take more risk in the process. Since not all of the costs can be legally protected, followers can easily freeride on the leader's efforts. While this freerider problem might be problematic from the viewpoint of protecting the investment incentives, the current analysis provides an alternative view that the asymmetric share of costs could improve the way the economy reaches consensus.

Of course, as can be seen from Proposition 5, it is not always welfare-improving to have a positive $c$. The norm which shifts more burden to the leader might work well either when the 
productivity dispersion is small or when both the productivity dispersion and the preference dispersion are large, e.g., in situations that are highly political in nature. In contrast, when the productivity dispersion is large but the preference dispersion is small, not enough people step up in equilibrium and the norm which imposes more cost to the leader aggravates the situation even worse. If the initial burden is too large, only the dependent types, who expect to derive large benefits, are willing to step up, including the type with weak preferences. Since the type with weak preferences have no strict preference over the alternatives, they just randomly pick one alternative and wait for others to join. The independent types follow the lead, but they include the type with strong preferences who has a strong stand as to which way to go and suffers from joining the alternative they dislike. As such, if the allocation of costs and benefits is not appropriate, the group cannot maximize the probability of successfully forming a network, thereby failing to pick an alternative favored by the majority.

\section{Conclusion}

Two players, with a potential conflict of interest, are asked to make a choice between two projects. They derive benefit only if they agree on the project to implement. In this context, we characterize the equilibrium timing of adoption as well as the efficient timing and show that the equilibrium timing is often different from the efficient timing in the presence of network externalities. Given this inefficiency result, we then show that the efficient timing can be implemented by a simple fee scheme which imposes fees on players contingent on the timing of adoption. The analysis gives an insight into why we often end up with inefficient consensus or standard, and suggests a potential role of social norms in resolving the coordination problem.

As a final note, the model is deliberately kept simple to illuminate the basic tradeoff. For this reason, there are several avenues to extend the current analysis. An obvious extension is to increase the number of players and time periods. The most general form along this line is perhaps a $T$ period model with $n$ players in which at least $m, n \geq m \geq 2$, players need to take the same side to yield any value. While the extension apparently makes the analysis more complicated and its underlying logic less transparent, we expect that the same principle applies for this more general setting as well because one can gain from expressing one's opinion early to lure other players to his side at a potential cost that he loses his chance to learn others' preferences. It is nonetheless of some interest to analyze this general model in future. 


\section{References}

[1] W. Brian Arthur. (1994) Inreasing Returns and Path Dependency in the Economy. Ann Arbor: University of Michigan.

[2] Marco Battaglini. (2005) "Sequential Voting with Abstention." Games and Economic Behavior, Vol. 51, pp. 445-463.

[3] Marco Battaglini, Rebecca Morton, Thomas Palfrey. (2007) "Efficiency, Equity, and Timing of Voting Mechanisms." American Political Science Review, Vol. 101, No. 3, pp 409-424.

[4] Janet M. Box-Steffensmeier, Laura W. Arnold, Christophis J. W. Zorn. "The Strategic Timing of Position Taking in Congress: A Study of the North American Free Trade Agreement." American Political Science Review, Vol. 91, No. 2, pp. 324-338.

[5] Gregory A. Caldeira and Christophis Zorn. (2004) "Strategic Timing, Position-Taking, and Impeachment in the House of Representatives." Political Research Quarterly, Vol. 57, No. 4, pp. 517-527.

[6] Steven Callander. (2007) "Bandwagons and Momentum in Sequential Voting." Review of Economic Studies, Vol. 74, pp. 653-684.

[7] Steven Callander. (2008) "Majority Rule When Voters Like to Win." Games and Economic Behavior, Vol. 64, pp. 393-420.

[8] Jay A. Conger and Rabindra N. Kanungo. (1998) Charismatic Leadership in Organizations, Thousand Oaks, CA: Sage Publications.

[9] Eddie Dekel and Michele Piccione. (2000) "Voting Procedures in Symmetric Binary Elections." Journal of Political Economy, Vol. 108, No. 1, pp. 34-55.

[10] Joseph Farrell and Carl Shapiro. (1988) "Dynamic Competition with Switching Costs." RAND Journal of Economics, Vol. 19, No. 1 (Spring, 1988), pp. 123-137.

[11] Joseph Farrell and Carl Shapiro. (1993) "The Dynamics of Bandwagons." in Problems of Coordination in Economic Activity, edited by James W. Friedman: Kluwer, 1993.

[12] Joseph Farrell and Garth Saloner. (1985) "Standardization, Compatibility, and Innovation." RAND Journal of Economics, Vol. 16, No. 1, pp. 70-83. 
[13] Joseph Farrell and Garth Saloner. (1986) "Installed Base and Compatibility: Innovation, Product Preannouncements, and Predation." American Economic Review, Vol. 76, No. 5, pp. 940-955.

[14] Michael L. Katz and Carl Shapiro. (1986) "Technology Adoption in the Presence of Network Externalities.” Journal of Political Economy, Vol. 94, No. 4, pp. 822-841.

[15] Michael L. Katz and Carl Shapiro. (1986) "Product Compatibility Choice in a Market with Technological Progress." Oxford Economic Papers, New Series, Vol. 38, Supplement: Strategic Behaviour and Industrial Competition, pp. 146-165.

[16] Robert J. Kauffman, Hsiangchu Lai, Chao-Tsung Ho. (2010) "Electronic Commerce Research and Applications." Electronic Commerce Research and Applications, 9, pp. 249-262.

[17] Ji-Ren Lee, Donald E. O’Neal, Mark W. Pruett, and Howard Thomas. (1995) "Planning for Dominance: A Strategic Perspective on the Emergence Process." R\&D Management, 25, pp. $3-26$.

[18] Marco Ottaviani and Peter Sorensen. (2001) "Information Aggregation in Debate: Who Should Speak First?" Journal of Public Economics, 81, pp. 393-421.

\section{Appendix A: Proofs}

Proof of Lemma 3: Let $U_{t}^{j}\left(r_{1}\right), j=D W, D S, I W, I S$, denote the expected payoff when a type $j$ player adopts in period $t$ for a given $r_{1}$, and let $\Delta U^{j}\left(r_{1}\right):=U_{1}^{j}\left(r_{1}\right)-U_{2}^{j}\left(r_{1}\right)$ denote the payoff difference for type $j$. The efficient order is obtained by ordering the types in terms of the payoff difference, where type $j$ is listed higher than type $j^{\prime}$ if $\Delta U^{j}\left(r_{1}\right)>\Delta U^{j^{\prime}}\left(r_{1}\right)$.

It is straightforward to compute the payoff difference for each type. It follows from (1) and (2) as well as the fact that $r_{1}+r_{2}=1$ for any efficient allocation that

$$
\begin{aligned}
& \Delta U^{D W}\left(r_{1}\right)=\frac{1-2 r_{1}}{2}\left(v_{H}-\frac{d}{2}\right), \\
& \Delta U^{D S}\left(r_{1}\right)=\frac{1-2 r_{1}}{2} v_{H}+\frac{r_{1}}{2} d, \\
& \Delta U^{I W}\left(r_{1}\right)=\frac{1-2 r_{1}}{2}\left(v_{L}-\frac{d}{2}\right), \\
& \Delta U^{I S}\left(r_{1}\right)=\frac{1-2 r_{1}}{2} v_{L}+\frac{r_{1}}{2} d .
\end{aligned}
$$


Simple algebra then establishes the lemma.

Proof of Proposition 2: Since $\Delta_{v}<\frac{d}{2}$, we can focus on the orders labeled as B and C in Lemmas 3. Given the orders, we need to choose the efficient value of $r_{1}$ which maximizes the total profit.

First consider the case where $q>\frac{1}{2}$. When $r_{1}=\frac{1}{2}$ and the efficient order is $\mathrm{C}$ in which the independent type with strong preferences and part of the dependent type with strong preferences adopt in the first period. The expected total payoff is

$$
\begin{aligned}
\pi\left(r_{1}\right)= & {\left[q(1-p)\left(1-\frac{r_{1}}{2}\right) v_{L}+\left(r_{1}-q(1-p)\right)\left(1-\frac{r_{1}}{2}\right) v_{H}\right] } \\
& +\left(q-r_{1}\right)\left(\frac{1}{2} v_{H}+\frac{r_{1}}{2}\left(v_{H}-d\right)\right) \\
& +(1-q)(1-p)\left(\frac{1}{2}+\frac{r_{1}}{2}\right)\left(v_{L}-\frac{d}{2}\right)+(1-q) p\left(\frac{1}{2}+\frac{r_{1}}{2}\right)\left(v_{H}-\frac{d}{2}\right) .
\end{aligned}
$$

It follows from this that

$$
\pi^{\prime}\left(r_{1}\right)=\frac{1}{2}\left[\left(1-r_{1}\right) v_{H}-r_{1}\left(v_{H}-d\right)\right]-\frac{1}{2}\left[r_{1} \bar{v}_{1}\left(r_{1}\right)-\left(1-r_{1}\right) \bar{v}_{2}\left(r_{1}\right)\right],
$$

where $\bar{v}_{1}\left(r_{1}\right)=\frac{1}{r_{1}}\left(q(1-p) v_{L}+\left(r_{1}-q(1-p)\right) v_{H}\right)$ is the expected value of those who adopt in the first period while assuming that the types with strong preferences work on the project they like, and $\bar{v}_{2}\left(r_{1}\right)=\frac{1}{1-r_{1}}\left[\left(q-r_{1}\right)\left(v_{H}-d\right)+(1-q)(1-p)\left(v_{L}-\frac{d}{2}\right)+(1-q) p\left(v_{H}-\frac{d}{2}\right)\right]$ is the expected value of those who adopt in the second period while assuming that the types with strong preferences work on the project they dislike. Note that when $r_{1}=\frac{1}{2}$, $\left(1-r_{1}\right) v_{H}-r_{1}\left(v_{H}-d\right)=\frac{d}{2}$ and $r_{1} \bar{v}_{1}-\left(1-r_{1}\right) \bar{v}_{2}<\frac{d}{2}$, so $\pi^{\prime}\left(r_{1}\right)>0$. Furthermore, for $r_{1} \in\left(\frac{1}{2}, q\right), \pi^{\prime}\left(r_{1}\right)$ decreases in $r_{1}$. For $r_{1}>q$, some proportion of the types with weak preferences also adopt in the first period, and we can show that $\pi^{\prime}\left(r_{1}\right)<0$ and $\pi^{\prime}\left(r_{1}\right)$ decreases in $r_{1}$. Therefore, we know that for $r_{1} \geq \frac{1}{2}$, the total payoff is maximized at some $r_{1} \in\left(\frac{1}{2}, p\right]$. For $r_{1} \in\left[1-q, \frac{1}{2}\right)$, the efficient order is $\mathrm{B}$, in which the dependent type with strong preferences and part of the independent type with strong preferences adopt in the first period. In this case,

$$
\pi^{\prime}\left(r_{1}\right)=\frac{1}{2}\left[\left(1-r_{1}\right) v_{L}-r_{1}\left(v_{L}-d\right)\right]-\frac{1}{2}\left[p_{1} \bar{v}_{1}\left(r_{1}\right)-\left(1-r_{1}\right) \bar{v}_{2}\left(r_{1}\right)\right]
$$

where

$$
\begin{gathered}
\bar{v}_{1}\left(r_{1}\right)=\frac{1}{r_{1}}\left(p q v_{H}+\left(r_{1}-p q\right) v_{L}\right) \\
\bar{v}_{2}\left(r_{1}\right)=\frac{1}{1-r_{1}}\left[\left(q-r_{1}\right)\left(v_{L}-d\right)+(1-q) p\left(v_{H}-\frac{d}{2}\right)+(1-q)(1-p)\left(v_{L}-\frac{d}{2}\right)\right] .
\end{gathered}
$$


When $r_{1}=\frac{1}{2},\left(1-r_{1}\right) v_{L}-r_{1}\left(v_{L}-d\right)=\frac{d}{2}$, and

$$
\begin{aligned}
r_{1} \bar{v}_{1}\left(r_{1}\right)-\left(1-r_{1}\right) \bar{v}_{2}\left(r_{1}\right)= & (1-q) p\left(v_{H}-\left(v_{H}-\frac{\Delta}{2}\right)\right)+(2 q-1) p\left(v_{H}-\left(v_{L}-\frac{d}{2}\right)\right) \\
& +((1-q)-p q)\left(v_{L}-\left(v_{L}-\frac{d}{2}\right)\right) \\
& +\left(\frac{1}{2}-(1-q)\right)\left(v_{L}-\left(v_{L}-d\right)\right)<\frac{d}{2},
\end{aligned}
$$

by Assumption 1. We can further show that $\pi^{\prime}\left(r_{1}\right)$ decreases in $r_{1}$ for $r_{1}<\frac{1}{2}$. Therefore, $r_{1}^{* *} \in\left(\frac{1}{2}, q\right]$.

Applying similar arguments, we can also prove that $r_{1}^{* *} \in\left[q, \frac{1}{2}\right)$ if $q<\frac{1}{2}$, and $r_{1}^{* *}=\frac{1}{2}$ if $q=\frac{1}{2}$.

Proof of Proposition 3: Suppose $q>\frac{1}{2}$. When $r_{1}=\frac{1}{2}$ and the efficient order is C, by $(5), \frac{\partial \pi^{\prime}\left(\frac{1}{2} ; v_{H}\right)}{\partial v_{H}}=\frac{1}{2}(1-p)(2 q-1)>0$, so $\pi^{\prime}\left(\frac{1}{2}\right)>0$ when $v_{H}$ is large enough. Furthermore, $\pi^{\prime \prime}\left(r_{1}\right) \geq-\left(2 v_{H}-d\right)$ for $r_{1}>\frac{1}{2}$, and we can find $u$ such that for $v_{H}>u$, $\pi^{\prime}\left(\frac{1}{2}\right)-\left(2 v_{H}-d\right) \frac{\frac{d}{2}}{2\left(v_{H}-v_{L}\right)}>0$, so for $v_{H}>u, \pi^{\prime}\left(r_{1}\right)>0$ for $r_{1} \in\left[\frac{1}{2}, \frac{v_{H}-v_{L}+\frac{d}{2}}{2\left(v_{H}-v_{L}\right)}\right]$, and $r_{1}^{* *}$ does not fall in $\left[\frac{1}{2}, \frac{v_{H}-v_{L}+\frac{d}{2}}{2\left(v_{H}-v_{L}\right)}\right]$. Similarly, we can show that when $v_{H}$ is large enough, $\pi^{\prime}\left(r_{1}\right)<0$ for $r_{1} \in\left[\frac{v_{H}-v_{L}-\frac{d}{2}}{2\left(v_{H}-v_{L}\right)}, \frac{1}{2}\right]$, so $r_{1}^{* *}$ does not fall in $\left[\frac{v_{H}-v_{L}-\frac{d}{2}}{2\left(v_{H}-v_{L}\right)}, \frac{1}{2}\right]$. Similar arguments can be applied to the case when $q<\frac{1}{2}$, so $r_{1}^{* *} \in\left(0, \frac{v_{H}-v_{L}-\frac{d}{2}}{2\left(v_{H}-v_{L}\right)}\right) \cup\left(\frac{v_{H}-v_{L}+\frac{\Delta}{2}}{2\left(v_{H}-v_{L}\right)}, 1\right)$ when $q \neq \frac{1}{2}$.

When $q=\frac{1}{2}$, for $r_{1} \in\left(\frac{1}{2}, \frac{1}{2}+(1-p)\left(1-p_{H}\right)\right)$, the types with strong preferences and part of the independent type with weak preferences adopt in the first period. When $v_{H}$ is large enough, $\frac{v_{H}-v_{L}+\frac{d}{2}}{2\left(v_{H}-v_{L}\right)}<\frac{1}{2}+(1-q)(1-p)$, and applying the same logic as before, $\pi^{\prime}\left(r_{1}\right)<0$ for $r_{1} \in\left(\frac{1}{2}, \frac{v_{H}-v_{L}+\frac{\Delta}{2}}{2\left(v_{H}-v_{L}\right)}\right)$, so $r_{1}^{* *}$ cannot fall in $\left(\frac{1}{2}, \frac{v_{H}-v_{L}+\frac{d}{2}}{2\left(v_{H}-v_{L}\right)}\right]$. Then we show that when $v_{H}$ is large, choosing $r_{1} \in\left(\frac{v_{H}-v_{L}+\frac{d}{2}}{2\left(v_{H}-v_{L}\right)}, 1\right)$ yields a higher total payoff than choosing $r_{1}=\frac{1}{2}$. Given $q=\frac{1}{2}, \frac{\partial \pi\left(\frac{1}{2} ; v_{H}\right)}{\partial v_{H}}=\frac{3}{4} p$. With $r_{1} \in\left(\max \left(\frac{v_{H}-v_{L}+\frac{\Delta}{2}}{2\left(v_{H}-v_{L}\right)}, 1-p\right), 1-(1-q) p\right)$, the independent types and some of the dependent type with strong preferences adopt in the first period, and $\frac{\partial \pi\left(r_{1} ; v_{H}\right)}{\partial v_{H}}=p\left(\frac{1}{2}+\frac{r_{1}}{2}\right)>\frac{3}{4} p$. Therefore, there exists $v$ such that if $v_{H}>v$, $\pi\left(r_{1}\right)>\pi\left(\frac{1}{2}\right)$, where $r_{1} \in\left(\frac{v_{H}-v_{L}+\frac{d}{2}}{2\left(v_{H}-v_{L}\right)}, 1\right)$. Similarly, we can show that when $v_{H}$ is large, choosing $r_{1} \in\left(0, \frac{v_{H}-v_{L}-\frac{d}{2}}{2\left(v_{H}-v_{L}\right)}\right)$ yields a higher total payoff than choosing $r_{1} \in\left[\frac{v_{H}-v_{L}-\frac{d}{2}}{2\left(v_{H}-v_{L}\right)}, \frac{1}{2}\right]$. So, $r_{1}^{* *} \in\left(0, \frac{v_{H}-v_{L}-\frac{d}{2}}{2\left(v_{H}-v_{L}\right)}\right) \cup\left(\frac{v_{H}-v_{L}+\frac{d}{2}}{2\left(v_{H}-v_{L}\right)}, 1\right)$ when $q=\frac{1}{2}$. 
Next we compare the payoffs when $r_{1} \in\left(0, \frac{v_{H}-v_{L}-\frac{d}{2}}{2\left(v_{H}-v_{L}\right)}\right)$ and when $r_{1} \in\left(\frac{v_{H}-v_{L}+\frac{d}{2}}{2\left(v_{H}-v_{L}\right)}, 1\right)$. By deriving $\pi^{\prime}\left(r_{1}\right)$ as in the proof of Proposition 2, we can show that as $v_{H}$ increases, if $r_{1}^{* *} \in\left(0, \frac{v_{H}-v_{L}-\frac{d}{2}}{2\left(v_{H}-v_{L}\right)}\right), r_{1}^{* *}$ converges to $\frac{1+p}{4} \in\left(\frac{1}{3}, \frac{5}{12}\right)$; if $r_{1}^{* *} \in\left(\frac{v_{H}-v_{L}+\frac{d}{2}}{2\left(v_{H}-v_{L}\right)}, 1\right), r_{1}^{* *}$ converges to $1-\frac{1+p}{4} \in\left(\frac{7}{12}, \frac{2}{3}\right)$. Therefore, given a small number $\epsilon>0$, there exists $v^{\prime}>v$ such that if $v_{H}>v^{\prime}, r_{1}^{* *}$ falls either in $\left(\frac{1+p}{4}-\epsilon, \frac{1+p}{4}+\epsilon\right)$ or in $\left(\frac{3-p}{4}-\epsilon, \frac{3-p}{4}+\epsilon\right)$. Suppose that $r_{1}^{* *} \in\left(\frac{1+p}{4}-\epsilon, \frac{1+p}{4}+\epsilon\right)$. By choosing $r_{1}^{\prime}=1-r_{1}^{* *}$, the expected total payoff increases by

$$
\begin{aligned}
& {\left[\left(q(1-p)+\max \left\{p q-r_{1}^{* *}, 0\right\}\right) r_{1}^{* *}-\max \left\{r_{1}^{* *}-p(1-q), 0\right\} r_{1}^{\prime}\right] d } \\
\simeq & {\left[\left(q(1-p)+\max \left\{p q-\frac{1+p}{4}, 0\right\}\right) \frac{1+p}{4}-\max \left\{\frac{1+p}{4}-p(1-q), 0\right\} \frac{3-p}{4}\right] d(.7) }
\end{aligned}
$$

When $q$ is small, (7) is positive, ${ }^{18}$ so choosing $r_{1}^{\prime}$ is better than choosing $r_{1}^{* *}$. Therefore, when $q$ is small enough, $r_{1} \in\left(\frac{v_{H}-v_{L}+\frac{d}{2}}{2\left(v_{H}-v_{L}\right)}, 1\right)$. Similarly, we can show that when $q$ is large enough, $r_{1} \in\left(0, \frac{v_{H}-v_{L}-\frac{d}{2}}{2\left(v_{H}-v_{L}\right)}\right)$.

Proof of Proposition 4: When $q<\frac{1}{2}, r_{1}^{*}=\frac{1}{2}$ by Proposition 1, and $r_{1}^{* *}<\frac{1}{2}$ by Proposition 2, so $r_{1}^{* *}<r_{1}^{*}$. For $q \geq \frac{1}{2}$, given $\pi^{\prime}(\cdot)$ defined in $(5),{ }^{19}$ let $\bar{r}(q)$ be such that $\pi^{\prime}(\bar{r}(q))=0$. For $q=\frac{1}{2}, \bar{r}(q)=\frac{2 v_{H}-\frac{3}{4} d}{4 v_{H}-2 d}>\frac{1}{2}$. Furthermore, $\bar{r}^{\prime}(q)=\frac{2(1-p)\left(v_{H}-v_{L}\right)-\frac{d}{2}}{4 v_{H}-2 d}$, and $\bar{r}(q) \in\left(\frac{1}{2}, \frac{v_{H}}{2 v_{H}-d}\right)$ for $q>\frac{1}{2}$. By Proposition 2 , for $q \geq \frac{1}{2}, r_{1}^{* *}=\bar{r}(q)$ if $\bar{r}(q) \leq q$, and $r_{1}^{* *}=q$ if $\bar{r}(q)>q$. By Proposition $1, r_{1}^{*}=q$ for $q \in\left(\frac{1}{2}, \frac{v_{H}}{2 v_{H}-d}\right]$. Therefore, when $q=\frac{1}{2}, r_{1}^{*}=r_{1}^{* *}$, and when $q=\frac{v_{H}}{2 v_{H}-d}, r_{1}^{*}<r_{1}^{* *}$. Since $r_{1}^{*}$ increases in $q$ linearly, and $\bar{r}(q)$ increases or decreases in $q$ linearly, there exists $\bar{q} \in\left(\frac{1}{2}, \frac{v_{H}}{2 v_{H}-d}\right]$ such that $r_{1}^{* *}=r_{1}^{*}$ if and only if $q \in\left[\frac{1}{2}, \bar{q}\right]$

Proof of Proposition 5: By comparing the results of Propositions 1 and 3, it is obvious that when $q \in\left(\bar{q}, \frac{2}{3}\right), r_{1}^{* *}<r^{*}$, and when $q \in\left(\frac{1}{3}, \underline{q}\right), r_{1}^{* *}>r^{*}$.

Proof of Proposition 6: When deriving the efficient order given $r_{1}$, we compare the expected payoffs when a type chooses to adopt in the first period and in the second period. A type with a larger payoff difference should be placed higher in the order. This is consistent with the players' incentive compatibility constraints. When a type is expected to get a higher payoff by adopting in the first period, he has a stronger incentive to adopt in the first period.

\footnotetext{
${ }^{18}$ For $q$ such that $p q-\frac{1+p}{4}<0, \frac{q(1-p)}{\max \left\{\frac{1+p}{4}-p(1-q), 0\right\}}$ decreases in $q$, so $(7)$ is positive when $q$ is small. For $q$ such that $p q-\frac{1+p}{4}>0, \frac{1+p}{4}-p(1-q)>0$, and $\frac{q(1-p)+p q-\frac{1+p}{4}}{\frac{1+p}{4}-p(1-q)}$ increases in $q$, but it can be shown that even when $q=\frac{2}{3},(7)$ is negative. Therefore, there exists $\bar{q}$ such that when $q<\bar{q},(7)$ is positive, and when $q>\bar{q}$, (7) is negative.

${ }^{19}$ Note that $\pi^{\prime \prime}(q)<0$.
} 
Therefore, to implement the efficient order given $r_{1}$, we only need to find out the type that is allocated to both periods, which we call the cutoff type for expositional purposes,${ }^{20}$ and find out $c$ that makes his payoffs in the two periods equal. Given $c$, the other types behave according to the efficient order.

To see how $c$ changes with $r_{1}$, let $r_{1}$ increase from $a$ to $a+\epsilon$, where $\epsilon>0$ is some small number. If $\frac{v_{H}-v_{L}-\frac{d}{2}}{2\left(v_{H}-v_{L}\right)}, \frac{1}{2}, \frac{v_{H}-v_{L}+\frac{d}{2}}{2\left(v_{H}-v_{L}\right)} \notin(a, a+\epsilon)$, then $r_{1}$ stays in the same region $(A, B, C$, or $D$ in Lemma 3$)$. If the cutoff type does not change, since $r_{1}$ increases, the type's first-period payoff decreases and the second-period payoff increases. Therefore, to make the two payoffs equal, $c$ must increase. If the cutoff type changes to the next type in the order, then we know that the difference between the two payoffs of this type is less than that of the old cutoff type, so $c$ must increase. Finally, if $b \in\left\{\frac{v_{H}-v_{L}-\frac{d}{2}}{2\left(v_{H}-v_{L}\right)}, \frac{1}{2}, \frac{v_{H}-v_{L}+\frac{d}{2}}{2\left(v_{H}-v_{L}\right)}\right\}$ and $b \in(a, a+\epsilon)$, when $r_{1}$ passes through $b$, the order changes. We already know that when $r_{1}$ increases from $a$ to $b$ or from $b$ to $a+\epsilon, c$ must increase as discussed just above. We thus need to see how $c$ should change when $r_{1}$ passes through $b$. If the cutoff type does not change, then nothing affects $c$. But if the cutoff type changes, it is implied that at $r_{1}=b$, the differences between the payoffs in the two periods of the new and the old cutoff types are the same, so $c$ is not affected either. This completes the proof that $c$ must increase as $r_{1}$ increases from $a$ to $a+\epsilon$.

\section{Appendix B: Computation of equilibrium}

In this appendix, we describe how we obtain the equilibrium allocation (the type-contingent probabilities of adoption) from the equilibrium order and $\left(r_{1}, r_{2}\right)$ as indicated in Proposition 1. First, by Lemma $1, r_{2}=1-r_{1}$, so $r_{1}$ is a sufficient statistic. Suppose that a player expects the other to adopt in the first period with probability $r$. For each given $r$, we can then check whether it is optimal to adopt or abstain in the first period for each type: (i) the dependent type with strong preferences adopt in the first period only if $R_{H} \geq r$; (ii) the independent type with strong preferences adopt only if $R_{L} \geq r$; (iii) the types with weak preferences adopt only if $0.5 \geq r$. From these incentive conditions, we can define a correspondence $F$ which maps this unconditional probability $r$ to the probability that a player adopts in the first period. The equilibrium $r_{1}$ is then obtained as a fixed point of $F$, i.e., $r_{1} \in F\left(r_{1}\right)$. See Figure 1 for a typical correspondence and an equilibrium. In this particular example, $r_{1}=q$

\footnotetext{
${ }^{20}$ For example, suppose that the efficient order is B. If $p q<r_{1}<p$, then the independent type with strong preferences is allocated to both periods. In this case, a fraction $\frac{r_{1}-p q}{q(1-p)}$ of the type chooses to adopt in the first period, while the remaining fraction chooses to adopt in the second period.
} 
where the types with strong preferences adopt in the first period and the types with weak preferences adopt in the second.

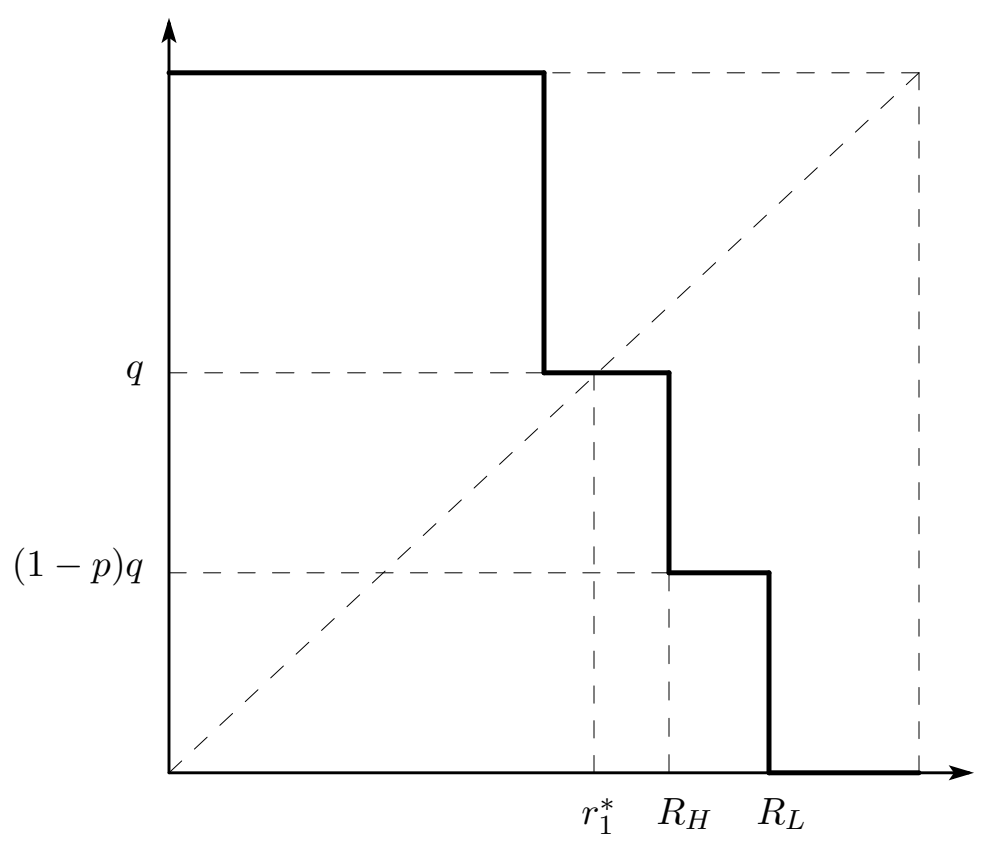

Figure 1: A typical correspondence and an equilibrium 